\title{
Modeling of Low-altitude Quasi-trapped Proton Fluxes at the Equatorial Inner Magnetosphere
}

\author{
A.A. Gusev, ${ }^{1,3}$ G.I. Pugacheva, ${ }^{2}$ U.B. Jayanthi ${ }^{1}$, and N. Schuch ${ }^{2}$ \\ ${ }^{1}$ Instituto Nacional de Pesquisas Espaciais/INPE, 122-970, São José dos Campus, SP, Brazil \\ ${ }^{2}$ Southern Regional Space Research Center; INPE, Santa Maria, Brazil \\ ${ }^{3}$ Space Research Institute/IKI, Russian Academy of Sciences, Moscow, Russia
}

Received on 15 January, 2003.

\begin{abstract}
A secondary proton radiation belt can be observed in the equatorial region between the upper atmosphere and the interior edge of the main radiation belt. It is thought that the protons appear there in a result of ionization of energetic neutral hydrogen atoms coming from the internal area of the traditional radiation belt where they were born in charge exchange collisions of the trapped protons with the cold hydrogen of the gecorona. The process of formation of this secondary belt is numerically simulated in this paper assuming this charge exchange-reionization mechanism. Standard models of the trapped radiation, of the atmosphere and geocorona were used to simulate the source and the exospheric media. Experimental data were used for charge transfer cross sections. Result of simulation agrees very good with the experimental observation.
\end{abstract}

\section{Introduction}

An experiment APEX - Monitoring of Alpha, Proton and Electron fluxes in the inner magnetosphere is being developed for the French-Brazilian Microsatellite (FBM) to be deployed in 2003. The satellite orbit with an inclination of $6-10^{\circ}$ and a height of $\approx 700 \mathrm{~km}$ will permit continuous monitoring of ion and electron fluxes essentially in the equatorial region of the innermost magnetosphere. The studies will be focused on the dynamical phenomena like fast radial diffusion and precipitation of the fluxes and their dependence on solar, magnetospheric and atmospheric conditions.

In the present work we consider the nature and characteristic of energetic proton environment in the equatorial region under the main radiation belt where the orbit of the FBM will pass. These protons are considered now also as a useful tool for observation of global magnetospheric dynamics.

The presence of a secondary radiation belt consisting of energetic protons and located in the interface region beneath the classic radiation belt and above the terrestrial atmosphere was established by Moritz [1] and Hovestadt [2]. They observed protons in the energy range of $0.25-1.65$ $\mathrm{MeV}$ between the altitudes from 400 to $1000 \mathrm{~km}$. Mizera and Blake [3] extended observation to lower energies measuring a spectrum of $12.5-500 \mathrm{keV}$ protons in the 400$470 \mathrm{~km}$ altitude range. Low-altitude rocket [4] and satellite measurements [1] indicate that the protons are concentrated at $90^{\circ}$ pitch angle with a magnitude independent of altitudes above $\sim 300 \mathrm{~km}$. More recently, Guzik et al. [5] discovered a strong altitude dependence in the flux of equatorial protons with energies from 0.6 to $9 \mathrm{MeV}$ at lower altitudes between 170 and $290 \mathrm{~km}$. We [6] examined the integral proton fluxes in the energy range $0.64-35 \mathrm{MeV}$ at $L$ values from 1.05 to 1.15 over a 3 -year period and observed abrupt flux enhancements of up to three orders of magnitude lasting for 1-3 days, and flux decreases of two orders of magnitude lasting a few monthes.

The observations reported were performed at low altitudes where geomagnetic drift shells are unclosed. Due to that we can formally regard the protons as quasi-trapped. In reality their lifetime is determined by charge exchange losses that is much shorter than the drift period. Due to that the fact that the drift shells are unclosed does not affect the flux magnitude of the protons, which is determined essentially by the same factors as inside the traditional radiation belt at closed drift lines.

Simple solid state detectors used mainly for detection of the protons in $k e V-M e V$ range in those experiments can only measure a sum of neutral and re-ionized fluxes. The neutral/re-ionized flux ratio strongly depends on the ion energy decreasing from $\sim 10$ at $10 \mathrm{keV}$ to $\sim 0.1$ at $100 \mathrm{keV}$. To select exactly neutrals one needs to eliminate proton background. It is usually done with constant magnets (see for example [4] ), that permits latitude-longitude mapping of the proton source. Contrary to that the simple telescopes measure a quasi-trapped flux that reflects the neutral flux averaged over a trajectory of Larmour rotation of the protons in the geomagnetic field.

A permanent, intense source needed to reproduce this population is identified as a charge exchange process of protons captured in the radiation belt and ring current region [1] as is illustrated in Fig. 1: Source trapped protons $\mathbf{H}^{+}$confined in the magnetosphere undergoes a charge exchange interaction with relatively cold $\left(\sim 1000^{\circ} \mathrm{K}\right)$ ambient neutral atom $\mathrm{A}^{0}$ e.g. $\mathbf{H}^{+}+\mathrm{A}^{0} \rightarrow \mathbf{H}^{0}+\mathrm{A}^{+}$(reaction $\mathbf{1}$ in Fig. ). After neutralization these protons appear as a fast non-thermal hydrogen neutral atoms $\mathbf{H}^{0}$ of the same energy and with the same velocity direction as those of a parent proton. Being 
neutral they are not affected or confined by the geomagnetic field and can travel in any direction. If they head earthward, there is a chance that they will collide with atmospheric neutral atoms and lose the electron in reaction of re-ionization $\mathbf{H}^{0}+\mathrm{A}^{0} \rightarrow \mathbf{H}^{+}+\mathrm{A}^{0}+\mathrm{e}^{-}$. (reaction 2 in Fig. 1) appearing now as positive ion of $\mathbf{H}^{+}$i.e. proton. If the angle be- tween velocity of the proton and geomagnetic line direction is sufficiently close to $90^{\circ}$ the proton can be trapped i.e. can perform geomagnetic drift along a line of the geomagnetic equator belonging to a definite $L$-shell. (Geomagnetic equator is defined as a plane crossing geomagnetic field lines in the point of minimum strength of the geomagnetic field.)

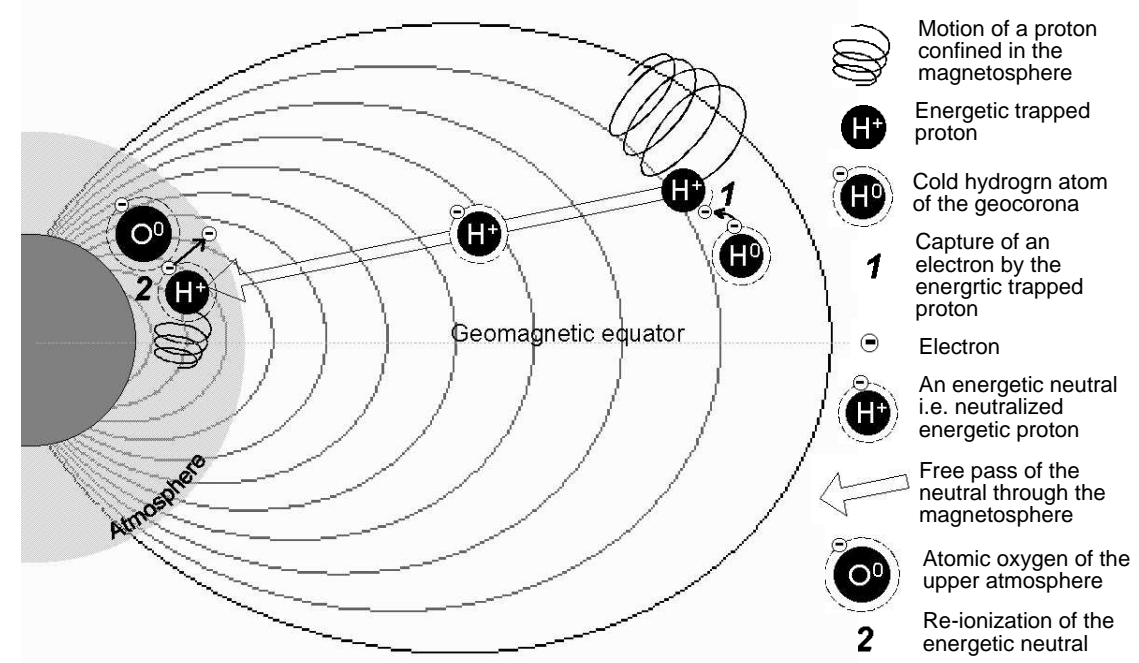

Figure 1. A scheme of formation of quasi-trapped proton population beneath the radiation belt. The geomagnetic field is approximated with field of a tilt shifted dipole. Geomagnetic equator is defined as a plane crossing geomagnetic field lines in the point of minimum strength of the geomagnetic field.

The mechanism described above provides a direct link between the radiation belt and the upper atmosphere at midlatitudes and low-latitudes. Because of their origin the characteristics of the neutral and quasi-trapped proton fluxes are closely related with those of the radiation belt proton population. It permits permanent monitoring the general dynamics of the ring current region and traditional proton radiation belt $[6,7]$ where direct measurements are still fragmental and rather complicated due to presence of intensive destructive radiation.

A noticeable variations of neutral and/or quasi-trapped fluxes correlated with geomagnetic storms were reported by Moritz [1], Mizera and Blake [3], Voss et al.[4], and Greenspan et al. [7]. Orsini et al. [8] simulated hydrogen and oxygen neutral fluxes basing on the data of AMPTEE/CCE measurements [9] and found that the neutral flux magnitude depends on the level of geomagnetic activity.

The transport of the neutral particles between the inner radiation belt and the upper atmosphere through the charge exchange and the following free flight across geomagnetic field lines was used for explanation of the observation of mid and low latitude precipitations [10-13]. Some estimations result to a flux of precipitating neutral hydrogen of 10 $\mathrm{keV}$ energy up to $2 \cdot 10^{5} 1 / \mathrm{cm}^{2} \mathrm{~s}$ sr $\mathrm{keV}$ during magnetic storm [14]. The penetrating neutral particles deposit considerable energy to the thermosphere heating the atmosphere. Intensive precipitation of $\mathrm{H}$ ions of $\mathrm{MeV}$ energy range were observed by Gusev et al. [6] when magnitude of precipitat- ing fluxes exceeded $10^{4} 1 / \mathrm{cm}^{2} s \mathrm{sr}$ in energy range $0.64-35$ $\mathrm{MeV}$.

The goal of the present work is simulation of quasitrapped proton fluxes in the assumption that they are produced in charge exchange-re-ionization process using the standard model of radiation belt. It will permit both to check the hypothesis of their origin and the radiation belt model itself. Until now no comparison of the physical model and experimental results has been done. This work is a part of the project of experimental study of energetic proton population in the innermost region of the magnetosphere (APEX) which will be performed on board a French-Brazilian satellite.

\section{Parameters of the model}

In the present work we only consider neutrals born with velocity vectors placed in the plane of the geomagnetic equator and directed to the center of the Earth. It means that we consider only the neutrals born by the trapped protons at the top of a geomagnetic field line with velocity vector placed in the meridian plane perpendicular to field line i.e. possessing a pitch angle of $90^{\circ}$.

The balance between sources and losses of the flux of the neutrals $F_{N}(E, x)$ of energy $E$ in the point located at the distance $x$ from the Earth is described as 


$$
\frac{d F_{N}(E, x)}{d x}=j_{T P}(E, x) P(E, x)-F_{N}(E, x) D(E, x)
$$

The first term in the right part of the equation describes production of the neutrals in the charge exchange reaction of trapped protons with exospheric cold atoms:

- $j_{T P}(E, x)$ is a directed flux of the trapped protons in $1 / \mathrm{cm}^{2}$ s sr $\mathrm{MeV}$,

- $P(E, x)=\sum_{i} \sigma_{10}^{i}(E) n^{i}(x) 1 / c m$,

- $\sigma_{10}^{i}(E) \mathrm{cm}^{2}$ is a cross section of electron capture from a cold neutral specie $i$ of the exosphere or atmosphere by energetic proton of energy $E$,

- $n^{i}(x) 1 / \mathrm{cm}^{3}$ is a density of neutral atoms of specie $i$.

The second term describes the losses of the energetic neutrals:

- $D(E, x)=\sum_{i} \sigma_{01}^{i}(E) n^{i}(x) 1 / \mathrm{cm}$

- $\sigma_{01}^{i}(E) n^{i} \mathrm{~cm}^{2}$ is a cross section of loss of electron by energetic neutral in collision with a neutral atom of specie $i$ of the exosphere or the upper atmosphere.
We take into account atomic and molecular hydrogen, helium, nitrogen and oxygen as main species constituting the atmosphere and exosphere.

To calculate a flux of energetic neutrals in a point of observation one needs to integrate the equation 1 along a path of view from the outer magnetospheric boundary to the point of observation.

Figure 2 presents the intensity of the source proton fluxes trapped in the plane of the geomagnetic equator. The unidirected flux magnitudes were derived from the standard model of the trapped radiation AP8 (see Acknowledgement) describing distribution of global (i.e. coming from all the direction of the $4 \pi$ solid angle) energetic proton fluxes in the magnetosphere of the Earth. The model contents a 3dimensional table of magnitudes of the integral global fluxes $J\left(>E, L, B_{P} / B_{0}\right)$ in $1 / \mathrm{cm}^{2} \mathrm{sec}$ in points characterized by proton energy $E, L$-shell number (i.e. distance from the geomagnetic dipole center of the top of the geomagnetic field line passing through the point ) and a ratio $B_{P} / B_{0}$ of magnetic field strength $B_{P}$ in the given point to magnetic field strength $B_{0}$ at the top of the same field line. One more part of the model is a FORTRAN code for access and interpolation of the data of the table. To convert global fluxes to unidirected ones $j_{\perp}\left(\left(>E, L, B_{P} / B_{0}\right)\right.$ perpendicular to the magnetic field line in the point with magnetic strength $B_{P}$ a following expression from Roederer [15] was used

$$
j_{\perp}\left(>E, L, B_{p} / B_{0}\right)=\frac{B_{p}^{3 / 2}}{2 \pi^{2}} \int_{B_{p}}^{B_{0}} \frac{d}{d B}\left[\frac{J\left(>E, L, B_{p} / B_{0}\right)}{B_{p}}\right] \frac{d B}{\left(B-B_{p}\right)^{1 / 2}}
$$

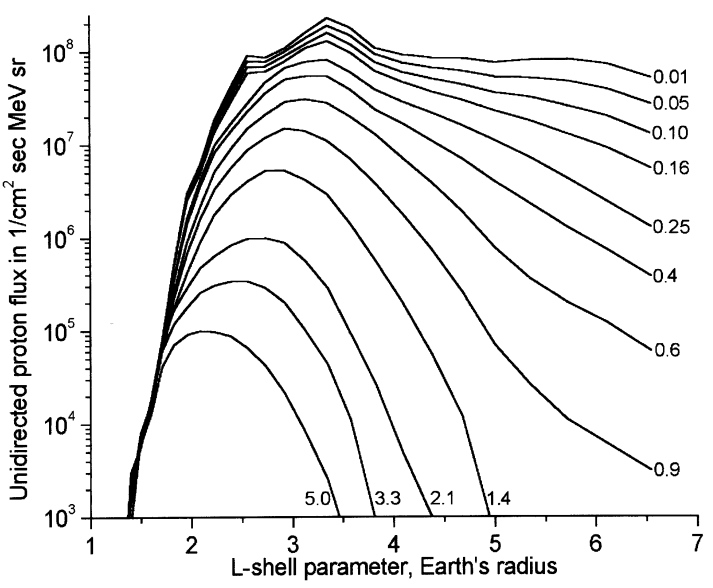

Figure 2. Intensity of the unidirected differential proton fluxes $j_{T P}(E, L)$ trapped in the equatorial plane. The numbers next to the curves mark proton energy in $\mathrm{MeV}$.
Integration is performed along the magnetic field line from the point $B_{p}$ to the point $B_{0}$. A problem of a singularity in the point $B=B_{p}$ was resolved by decreasing the integration step in the singularity vicinity until reaching a desirable convergence.

The procedure was performed for the each point of the AP8 table and a global flux value in the point was substituted with a value of the related unidirected flux. It permits to implement the same FORTRAN code of access and interpolation both for extracting and interpolation the global and the unidirected fluxes. Thus this new table can be considered as a model of the unidirected trapped proton fluxes.

Figure 3 demonstrates altitude dependences of densities of the most abundant atmospheric species. The data beneath $1000 \mathrm{~km}$ were taken from MSIS-E-90 atmospheric model [16]. The population of the neutral hydrogen above $1000 \mathrm{~km}$ for the temperature of $1024^{\circ} \mathrm{K}$ was simulated by 


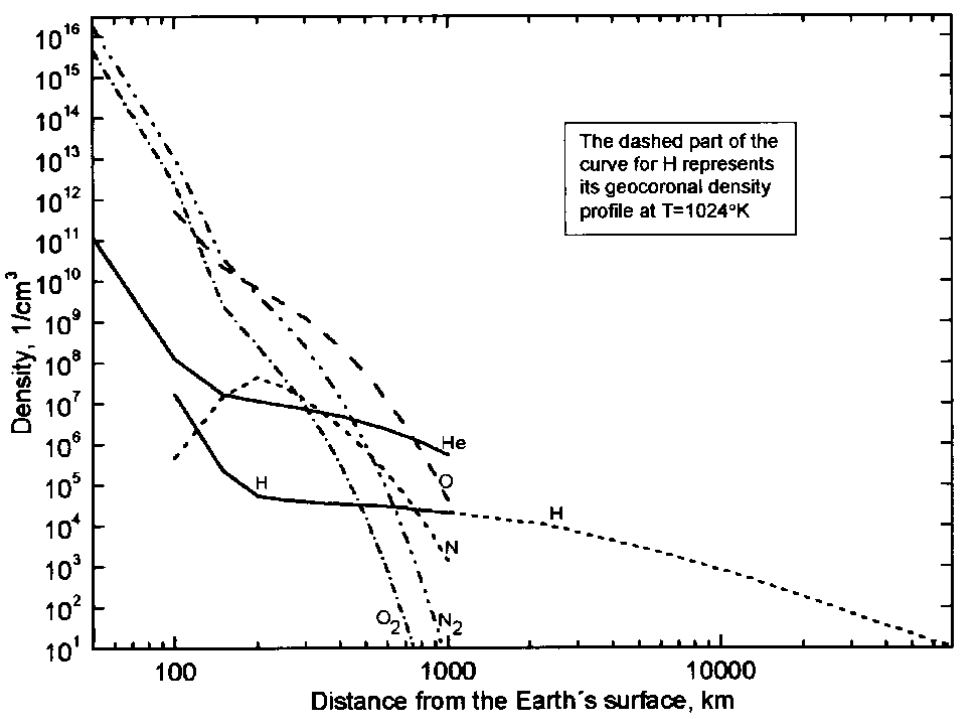

Figure 3. Atmospheric and exospheric densities according MSISE-90 model and Chamberlen geocoronal model.

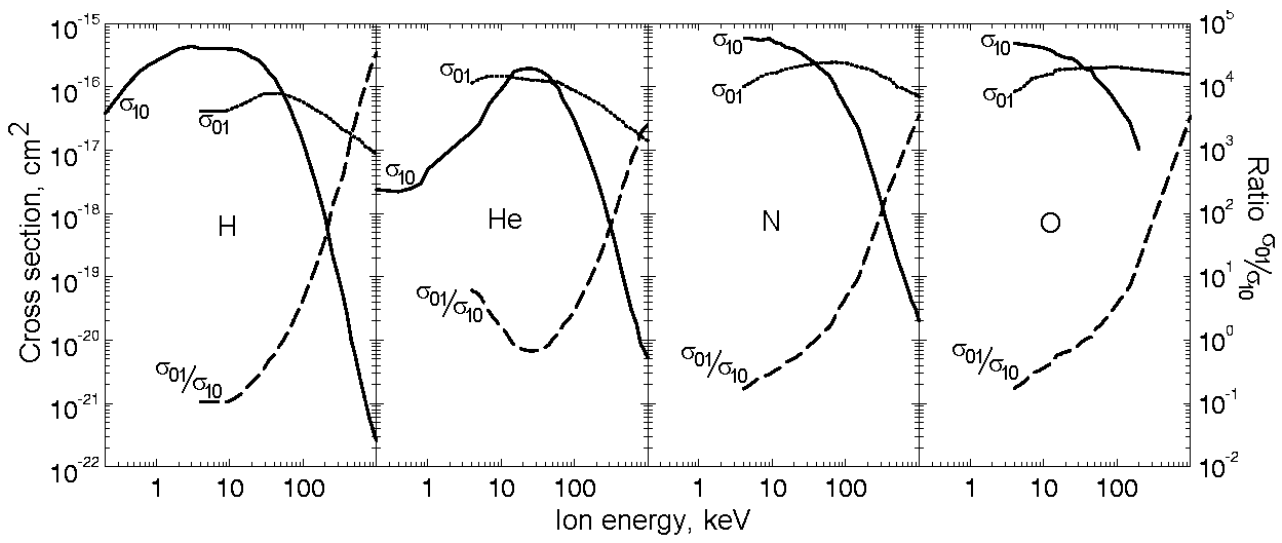

Figure 4. Charge transfer cross section for hydrogen ions according to Allison, [1958]. $\sigma_{10}$ - charge exchange cross section i.e. that of electron capture by energetic proton; $\sigma_{01}$ - ionization cross section i.e. that of loss of electron by an energetic neutral.

Rairden et al. [17] according a spherically symmetric geocoronal model of Chamberlen [18]. (Geocorona is a tenuous cloud of exospheric neutral hydrogen enveloping the Earth. It can be observed as a faint glow around our planet, produced in resonant scattering of solar Lyman alpha radiation.)

Figure 4 shows energy dependences of charge transfer cross section for the most important atomic atmospheric constituents measured by Allison [19]. To obtain the molecular cross sections for the energies $>1 \mathrm{keV}$ the atomic ones must be multiplied by the factor of 2 [20].

The only neutral specie present in significant numbers in the Earth's magnetosphere at the altitudes of the confinement region is atomic hydrogen. Due to that the reaction $\mathbf{H}^{+}$ $+\mathrm{H}^{0} \rightarrow \mathbf{H}^{0}+\mathrm{H}^{+}$is considered as a main source of energetic $\mathbf{H}^{0}$. On the other hand, the same mechanism of charge exchange is an important mechanism of losses of ions constituting the classical radiation belt (see for example [21] ).

One can see that charge exchange cross section $\sigma_{10}$ rapidly decreases with the energy for proton energy exceeding $10 \mathrm{keV}$. Due to that the resulting spectrum of the neutrals is significantly steeper than the parent trapped proton spectrum.

After a free flight across the geomagnetic field an energetic neutral produced with a velocity directed to the Earth reaches the residual atmosphere. It can be re-ionized there in collision with atomic constituents of the upper atmosphere. Being charged the re-ionized proton becomes to be captured again by the geomagnetic field and drifts along the geomagnetic equator line of the same $L$-shell at which they were produced in the re-ionization collision. The altitude of the geomagnetic equator line, along which the proton drifts, varies because of shift of the geomagnetic dipole. As a result the innermost geomagnetic equator lines are not closed i.e. they partially pass through the denser atmospheric layers beneath $\sim 100 \mathrm{~km}$. Descending down to these altitudes the protons die due to Coulomb scattering and neutraliza- 
tion. No new protons are produced there because the parent neutral flux is exhausted after passage through the above atmospheric matter. The other part of the re-ionized protons trapped at higher $L$-shell can perform noticeable number of turns around the Earth until loss their energy in Coulomb scattering or suffer neutralization collisions in the residual atmosphere. But there they can not be distinguished from the parent protons of the radiation belt.

The magnitude of the re-ionized proton flux during the geomagnetic capture is determined by the balance between the source (i.e. ionization of the neutral flux) and losses due to neutralization and Coulomb scattering. Fig. 5 demonstrate mean characteristic times of the processes involved.

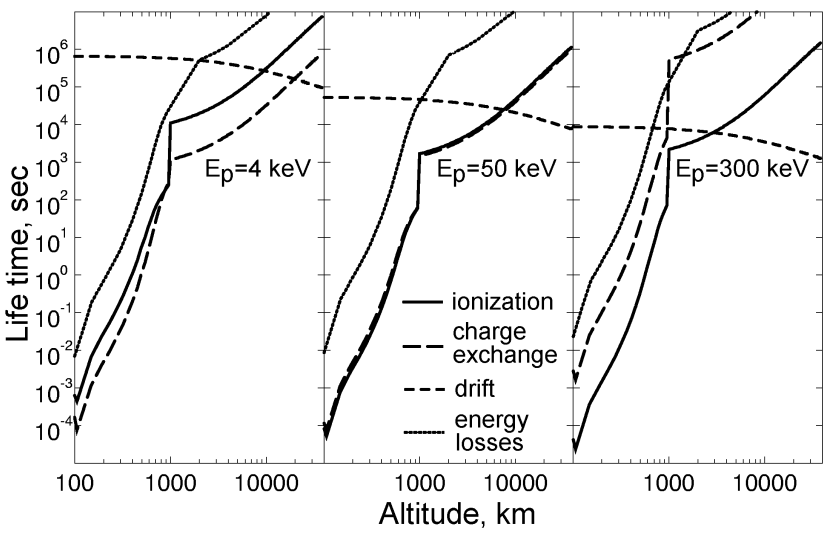

Figure 5. Character times of the processes involved in formation of the neutral and quasi-trapped proton fluxes: re-ionization (ionization), neutralization (charge exchange), Coulomb scattering (energy losses). Lifetime until Coulomb scattering is defined as time until losses of $30 \%$ of the initial energy of the proton.

One can see that lifetime until Coulomb scattering exceeds that until neutralization by about one order of magnitude at practically all the altitudes. However a real lifetime can be different because the altitude of the geomagnetic equator along which the proton drifts also varies. If the proton drifts sufficiently rapid its lifetime will be determined by the losses at the smallest altitude of the geomagnetic equator, where the atmospheric density is higher. On the unclosed drift trajectories i.e. on those with the minimal altitude $<100 \mathrm{~km}$, the proton lifetime is determined by the time which the proton spends at higher altitudes. It is approximately equal to the proton drift period around the Earth shown also in Fig. 5. It is also one order of magnitude higher compared to the neutralization lifetime for the altitudes $<1000 \mathrm{~km}$. At higher altitudes the drift motion does not result to particle losses because of small exospheric density. Due to that the corresponding lifetime is also incomparatively higher than those of the other processes. It means that a quasi-trapped proton dies close to the point where it was produced in re-ionization collision.

In reality the drift period is determined not by a local altitude as the Fig. 5 shows, but by an $L$-shell crossing a given altitude that depends on a longitude. Thus some range $\Delta L$ of $L$ relates with a selected altitude. It is determined by a distance of $\approx 5 \cdot 10^{2} \mathrm{~km}$ between the centers of the
Earth and the geomagnetic dipole. $\Delta L=5 \cdot 10^{2} / R_{\text {Earth }}=$ $5 \cdot 10^{3} / 6371.2 \approx 0.08$. Because a drift period is proportional to $1 / L$ and $\Delta L$ is sufficiently small a corresponding relative drift period range is less than 0.08 for $L>1$, i.e. does not practically differ from those shown at Fig. 5.

Thus the quasi-trapped proton lifetime is determined exclusively by the lifetime until charge exchange i.e. neutralization. In this case the number of emerging protons $F_{N}(E, x) \times D(E, x)$ is equal to the number of the protons vanishing due to neutralization $F_{P}(E, x) \times P(E, x)$ : $F_{N}(E, x) D(E, x)=F_{P}(E, x) P(E, x)$. As a result

$$
F_{P}(E, x)=F_{N}(E, x) D(E, x) / P(E, x)
$$

Depending on the energy the $D / P$ ratio can be both less and more than 1. Due to that the trapped proton flux can be both higher and lower in comparison with the parent neutral flux. The $D / P$ ratio is also shown in the Figure 4 . For the energies higher than $640 \mathrm{keV}$ the re-ionized flux is at least 1000 times higher than the neutral one i.e. the summarized flux of neutral and positive $\mathrm{H}$ ions consists practically only of re-ionized protons. That was experimentally confirmed by the measurements on board the OHZORA satellite [6].

\section{Results}

Differential equation (1) describing the charge exchange-re-ionization process with the parameters described above was simulated numerically using the RungeKutta method of the fourth order.

Figure 6 presents the radial dependence of the neutral fluxes for various energies of atoms. One can see that the flux saturates after the $L \approx 2$, remaining constant down to at least $L \approx 1.05$ and after that rapidly decreases due to losses in the upper atmosphere. This result is in qualitative agreement with the observed altitude dependence of the flux at low altitudes [5].

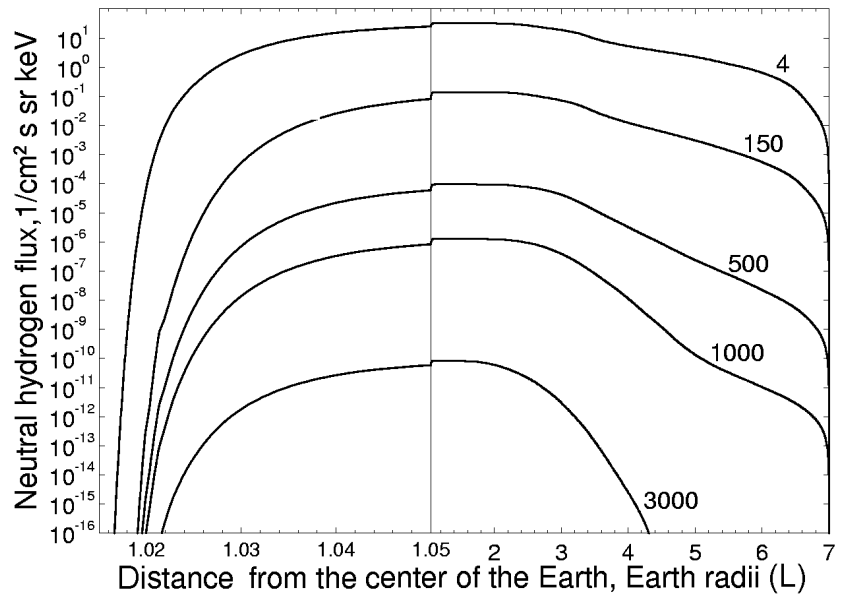

Figure 6. Radial dependence of the flux of the neutrals generated by the protons of the radiation belt. Numbers next the curves marks energy of neutrals in $\mathrm{keV}$.

The minimal altitude of the geomagnetic equator line of $L \approx 2$ is about $5 \cdot 10^{3} \mathrm{~km}$ and the maximal one of $L \approx 1.05$ 
is $\approx 300 \mathrm{~km}$. Thus the processes of accumulation and losses of the neutral flux are very well separated spatially. Due to that the equation (1) could be also separated for two different equation: the first describing neutral flux accumulation due to charge-exchange at $L>2$ and the second one describing neutral flux losses at the altitudes $<300 \mathrm{~km}$.

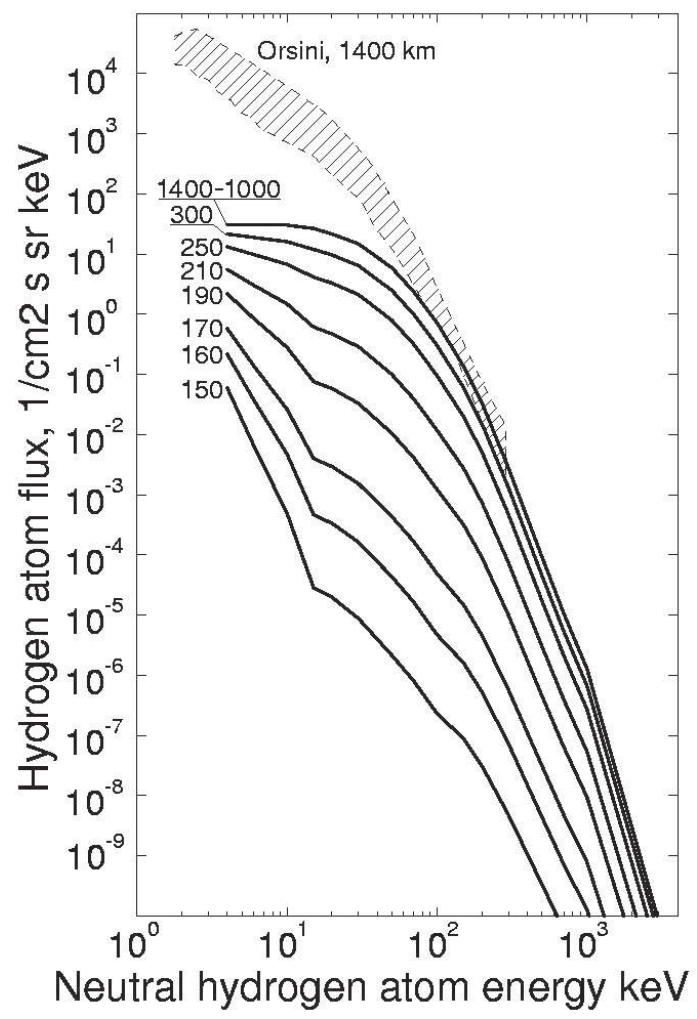

Figure 7. The spectra of the hydrogen neutrals at various altitudes. The numbers next to the curves marks the altitude above the Earth's surface in kilometers.

Figure 7 shows spectra of the neutrals at various altitudes. The results of the simulation above $1000 \mathrm{~km}$ is compared with the simulation of Orsini et al [8] based on the experimental data of the AMPTE/CCE CHEM experiment [9]. For the energies $<50 \mathrm{keV}$ the result of our simulation is significantly lower in comparison with that of Orsini. The reason of that is that the AMPTE/CCE CHEM experiment detected significantly higher fluxes of the $<50 \mathrm{keV}$ trapped protons than those constituting the AP- 8 model.

Altitude dependences of relative input produced by the trapped protons to the flux of the neutrals of various energies are shown in Fig. 8. Comparing it with the Fig. 2 one can note that the effectiveness of the trapped proton source for neutral production decreases with altitude due to decreasing density of the target exospheric hydrogen. Thus the neutral flux is mainly formatted by the protons of the more inner $(L<3)$ area of the magnetosphere.

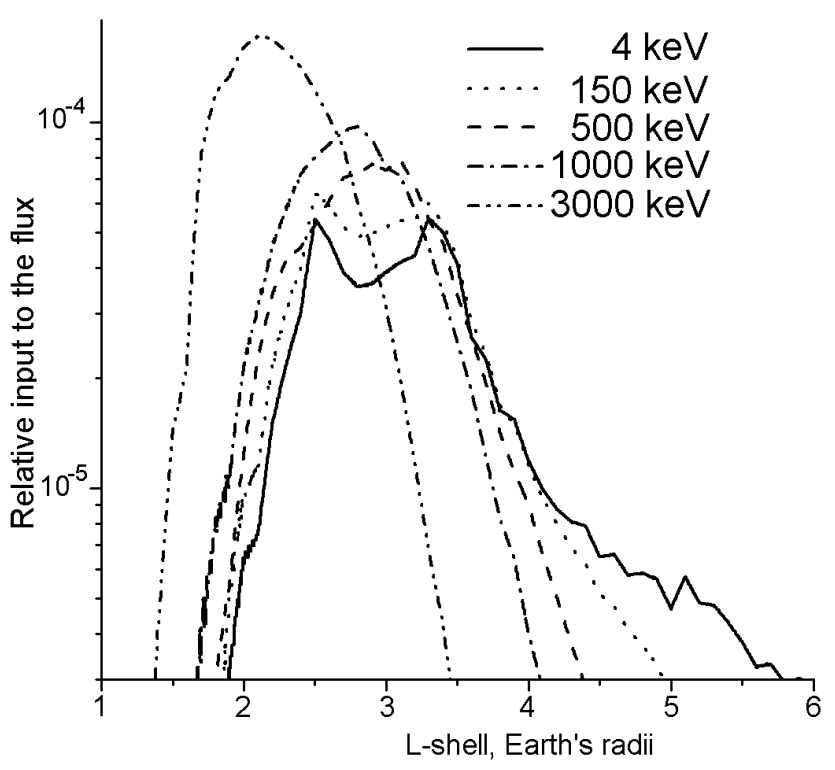

Figure 8. Altitude dependence of relative input produced by the trapped proton fluxes of various energies.

Figure 9 shows a flux of the re-ionized hydrogen ions calculated according to the equation 2. An abrupt changes in the curves at the altitude of $1000 \mathrm{~km}$ is a result of to the limitations of the atmospheric model used, which gives the gas concentrations only up to $1000 \mathrm{~km}$ (see Fig. 3). The geocorona model used for the higher altitudes contains no date for the elements heavier than hydrogen. Due to that the result from $1000 \mathrm{~km}$ up to $\sim 4000 \mathrm{~km}$ is not quite correct.

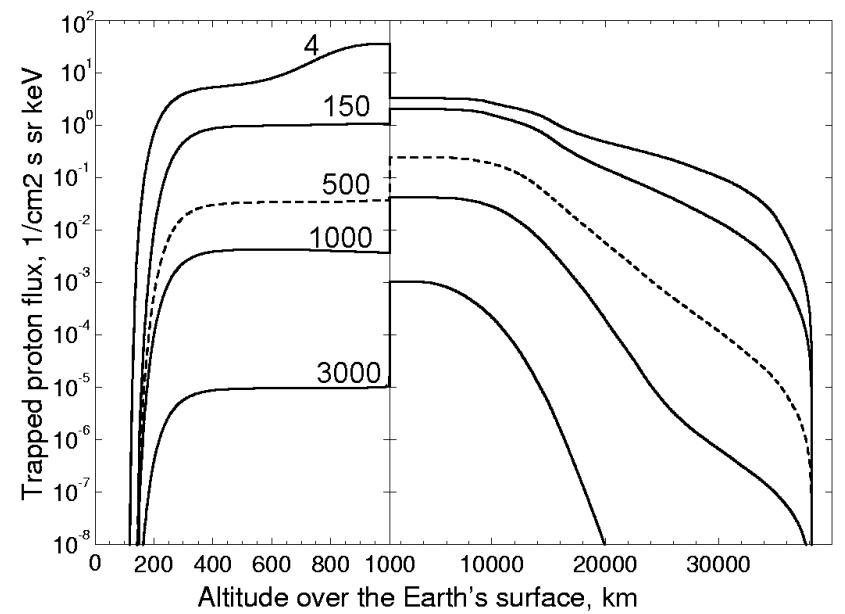

Figure 9. Dependence of the intensity of the re-ionized hydrogen ions (i.e. protons) versus altitude. The numbers next to the curves marks the ion energies. An abrupt bound of the simulated flux at the altitude of $1000 \mathrm{~km}$ is caused by incompleteness of the exospheric model used for the altitudes exceeding $1000 \mathrm{~km}$.

One can see that the proton population originated in neutralization-re-ionization process occupies a vast region of the magnetosphere comparable with that populated with the traditional trapped protons brought to the magnetosphere by radial diffusion. Thus it is not quite correct to speak that the neutralized-re-ionized protons exist only beneath the traditional radiation belt. In reality they can only be 
observed in that region where the intensity of the diffusive source of the inner radiation belt is already too low compared to the neutralization losses.

The results of the simulation are compared with experimental data in Fig. 10. The simulated flux agrees very well with experimental results including Orsini et al., [8]. To get the proton flux the neutral spectrum obtained in the latter inside the radiation belt was multiplied by the factor $D / P$ for the altitude of $1000 \mathrm{~km}$.

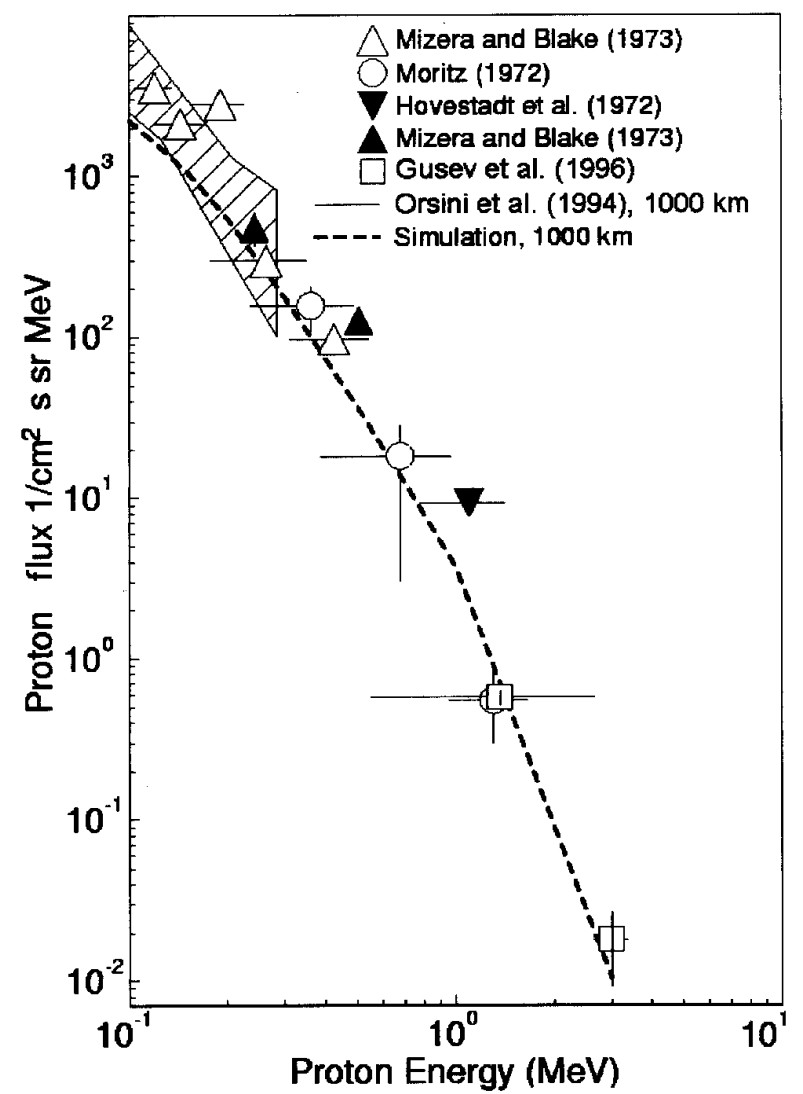

Figure 10. Comparison of the simulated result with experimental data.

\section{Conclusion}

We have numerically simulated the fluxes of the energetic neutral hydrogen atoms and quasi-trapped protons assuming their origin in charge-transfer reactions of the protons of the traditional radiation belt with constituencies of the exosphere and atmosphere. Standard models describing proton fluxes of the radiation belts, the atmospheric composition and the geocorona were used. It is shown that these protons occupy a vast region of the magnetosphere comparable with that populated with the protons of the traditional radiation belt. The shape and the absolute values of the simulated quasi-trapped proton spectra agree well with all the set of the experimental results. That is one more confirmation of correctness of the hypothesis of their origin and adequateness of the current atmospheric and magnetospheric models. The results presented here indicate to a potential of the quasitrapped proton observations for monitoring global dynamics of the inner atmosphere.

\section{Acknowledgments}

This work has been supported by grants from CNPq (A.A. Gusev) and FAPERGS (G.I. Pugacheva). The model AP8 was obtained from the web pages of NASA's National Space Science Data Center.

\section{References}

[1] J. Moritz, Z. Geophys. 38, 701 (1972).

[2] D. Hovestadt, B. Hausler, and M, Scholer, Phys. Rev. Lett. 28, 1340 (1972).

[3] P. F. Mizera, and J. B. Blake, J. Geophys. Res, 78, 1058 (1973).

[4] H. D. Voss and L. G. Smith, COSPAR Adv. Space Explor. 8, 131 (1979).

[5] T. G. Guzik, M. A. Miah, J. W.Mitchel, and J.P. Wefel, J. Geophys. Res, 94, 145 (1989).

[6] A. A. Gusev, T. Kohno, W. N. Spjeldvik, I. M. Martin, G. I. Pugacheva, and Turtelli Jr., J. Geophys. Res, 101, 19659 (1996).

[7] M. E. Greenspan, G. M. Mason, and J. E. Mazur, J. Geophys. Res, 104, 19911 (1999).

[8] S. Orsini, I.A. Daglis, M. Candidi, K.C. Hsieh, S. Livi, and Wilken, J. Geophys.Res, 99, 13489 (1994).

[9] G. Gloeckler, F. M. Ipavich, W. Studemann., B. Wilken, D. C. Hamilton, G. Kremser, D. Hovestadt, F. Gliem, R. A. Lundgren, W. Rieck, E. O. Tums, J. C. Cain, L. S. Masung, W. Weiss, and P. Winterhof charge-energy-mass (CHEM) spectrometer for $0.3-300 \mathrm{keV} / \mathrm{e}$ ions on the AMPTE/CCE, IEEE Transactions on geoscience and remote sensing, 23, 234 (1985).

[10] B. A. Tinsley, J. Atmos. Terr. Phys. 43, 617 (1981).

[11] B. A. Tinsley, R. P. Rhorbaugh, H. Rassoul, E. S. Barker, A. L. Cochran, W. D. Cochran, B. J. Wills, D. W. Wills, and D. Slater, Geophys. Res. Lett. 11, 572 (1984).

[12] B.A. Tinsley, R. P. Rhorbaugh, H. Rassoul, Y. Sahai, N.R. Teixeira, and D. Slater, J. Geophys. Res. 91, 11257 (1986a).

[13] B. A. Tinsley, R. R. Hodges, Jr., and R. P. Rhorbaugh, H. Rassoul, J. Geophys. Res. 91, 13631 (1996b).

[14] W. Bernstein, G.T. Inouye, N.L. Sanders, and R. L. Wax, J. Geophys. Res. 74, 3601 (1969).

[15] Roederer J. G., Dynamics of geomagnetically trapped radiation, Springer-Verlag, Heidelberg-New York, (1970).

[16] A. E. Hedin, J. Geophys. Res. 96, 1159 (1991).

[17] R. L. Rairden., L. A. Frank, and J. D. Craven, J. Geophys. Res, 91, 13613 (1986).

[18] J. W. Chamberlen, Planet Space Sci, 11, 901 (1963).

[19] S. K. Allison, Rev. Mod. Phys. 30, 1137 (1958).

[20] K. L. Riesselman, W. Anderson, L. Durand, and C. J. Anderson, Phys. Rev. A, 43, 5934 (1991).

[21] G. I. Pugacheva, A.A. Gusev, U. B. Jayanthi, I. M. Martin, and W. N. Spjeldvik, FEM code simulation of the magnetospheric proton fluxes, Adv. Space Res. 28, 12 (2001). 